Irene Bertschek

\title{
Digitalisierung - der Corona-Impfstoff für die Wirtschaft
}

Die Corona-Pandemie hinterlässt ihre Spuren in der Wirtschaft. Diese fallen je nach Branche recht unterschiedlich aus. Konsumnahe Dienstleistungsbranchen, in denen die Nähe zum Kunden wesentlich für die Erbringung ihrer Dienste ist, hat es besonders schwer getroffen. Dazu zählen Gastronomie und Beherbergung, Events und Veranstaltungen, Touristik und Sport sowie Wellness, Friseure und Kosmetik. Unter den Soloselbständigen in diesen Branchen mussten etwa neun von zehn Befragten Umsatzeinbußen verkraften, die über $75 \%$ lagen (Bertschek und Erdsiek, 2020). Während es in einigen Branchen langsam wieder aufwärts geht, beispielsweise in der deutschen Tourismusbranche, die davon profitiert, dass es zahlreiche Bundesbürger bevorzugen, nicht das Land zu verlassen, ist in anderen Branchen wie der Kulturwirtschaft kaum Licht am Ende des Tunnels erkennbar, da Kultur z.B. in Form von Konzerten oder Theateraufführungen in der Regel in größeren Gruppen konsumiert wird.

Eine im Juni 2020 durchgeführte Befragung der Kreditanstalt für Wiederaufbau KfW (Schwarz und Gerstenberger, 2020) im deutschen Mittelstand zeigt, dass wissensintensive Dienstleistungsunternehmen (67 \%) häufiger Umsatzeinbußen hinnehmen müssen als Unternehmen im verarbeitenden Gewerbe ( $60 \%$ ), ebenso kleinere Mittelständler mit bis zu zehn Beschäftigten (62\%) häufiger als größere mit mehr als zehn Beschäftigten (51\%). Die Baubranche kommt insgesamt relativ gut weg, hier kämpfen nur knapp ein Drittel der Unternehmen mit Umsatzeinbußen.

Es hat sich gezeigt, dass folgende drei Faktoren für Unternehmen entscheidend waren, um sich während des Lockdowns über Wasser zu halten: erstens, die Fähigkeit flexibel auf den Nachfragerückgang nach Produkten und Diensten oder auf Lieferengpässe zu reagieren und gegebenenfalls auf das Angebot anderer Produkte und Dienste umzustellen; zweitens, die Möglichkeit Distanz zu wahren, z. B. durch den Einsatz von Robotern in der Produktion und Logistik oder durch die Einführung oder Ausweitung von Homeoffice und Videokommunikation, und drittens, die Option für (potenzielle) Kunden und Kooperationspartner sichtbar zu bleiben.

(c) Der/die Autor(en) 2020. Open Access: Dieser Artikel wird unter der Creative Commons Namensnennung 4.0 International Lizenz (https:// creativecommons.org/licenses/by/4.0/deed.de) veröffentlicht.

Open Access wird durch die ZBW - Leibniz-Informationszentrum Wirtschaft gefördert.

\section{Die Corona-Krise beschleunigt die Digitalisierung}

Alle drei Überlebensstrategien werden durch die Digitalisierung begünstigt. Unter den Soloselbständigen hat durch die Krise etwa jeder Dritte Befragte bei der Digitalisierung zugelegt (Bertschek und Erdsiek, 2020). Besonders hervorzuheben sind hierbei die Bereiche Training und Schule, Gesundheit, Soziale Arbeit sowie stationärer Handel, wie in Tabelle 1 dargestellt. Ökonometrische Schätzungen zeigen zudem, dass ein Digitalisierungsschub tendenziell stärker stattgefunden hat bei Soloselbständigen, die weiblich oder vergleichsweise jung sind, die im Homeoffice arbeiten oder einen Hochschulabschluss haben. Während des Lockdowns waren zum Zeitpunkt der Befragung $73 \%$ der Soloselbständigen mit einem sehr niedrigen Digitalisierungsgrad des Angebots nicht mehr in der Lage, die eigene Tätigkeit auszuüben. Unter den sehr hoch digitalisierten Soloselbständigen waren es dagegen nur $28 \%$. Digitalisierte Soloselbständige erweisen sich also als deutlich krisenresistenter und leiden seltener unter den negativen Konsequenzen der Pandemie.

Ein krisenstabilisierender Effekt der Digitalisierung konnte bereits während der Finanz- und Wirtschaftskrise 2008/2009 ausgemacht werden, wie eine ökonometrische Analyse mittels eines Differenz-von-DifferenzenAnsatzes mit Daten zu zwölf europäischen Ländern und sieben Branchen zeigt (Bertschek et al., 2019). Bei Unternehmen, die hoch digitalisiert waren, nahm das Produktivitätswachstum während der Krise um 0,5 Prozentpunkte ab, bei gering digitalisierten Unternehmen sank es dagegen um 2,3 Prozentpunkte. Gleichzeitig gelang es höher digitalisierten Unternehmen, insbesondere im Dienstleistungssektor, eher Prozessinnovationen umzusetzen.

Prof. Dr. Irene Bertschek leitet den Forschungsbereich Digitale Ökonomie am ZEW - Leibniz-Zentrum für Europäische Wirtschaftsforschung in Mannheim und ist Professorin für Ökonomie der Digitalisierung an der Justus-Liebig-Universität Gießen. 
Tabelle 1

Hoher Digitalisierungsgrad vor der Krise und Veränderungen durch die Krise nach Branchen in \% der befragten Soloselbständigen

\begin{tabular}{|c|c|c|c|c|c|c|}
\hline & \multicolumn{3}{|c|}{ Hoher Digitalisierungsgrad vor der Krise } & \multicolumn{3}{|c|}{ Digitalisierungsgrad durch die Krise erhöht } \\
\hline & $\begin{array}{l}\text { Produkte/ } \\
\text { Dienstleistungen }\end{array}$ & $\begin{array}{l}\text { interne } \\
\text { Geschäfts- } \\
\text { prozesse }\end{array}$ & $\begin{array}{l}\text { Kundenbera- } \\
\text { tung/Vertrieb }\end{array}$ & $\begin{array}{l}\text { Produkte/ } \\
\text { Dienstleistungen }\end{array}$ & $\begin{array}{l}\text { interne } \\
\text { Geschäfts- } \\
\text { prozesse }\end{array}$ & $\begin{array}{l}\text { Kundenbera- } \\
\text { tung/Vertrieb }\end{array}$ \\
\hline IT-, Software-, Web-Dienstleistung & 87 & 83 & 63 & 16 & 12 & 22 \\
\hline Lektorat, Dolmetscher, Übersetzer & 67 & 67 & 58 & 17 & 7 & 11 \\
\hline Journalisten oder Public Relations & 65 & 67 & 46 & 14 & 14 & 19 \\
\hline Onlinehandel & 64 & 68 & 70 & 9 & 3 & 3 \\
\hline Ingenieure und Architekten & 58 & 62 & 36 & 13 & 12 & 19 \\
\hline Unternehmensnahe Dienstleistungen & 54 & 64 & 42 & 27 & 15 & 33 \\
\hline Finanzdienstleistungen/Versicherungen & 48 & 62 & 31 & 34 & 25 & 52 \\
\hline Event- und Veranstaltungsbranche & 27 & 49 & 32 & 9 & 7 & 10 \\
\hline Kulturschaffende, Film, Fotografie & 25 & 38 & 27 & 30 & 18 & 22 \\
\hline Touristik, Sport und Freizeit & 20 & 33 & 32 & 21 & 14 & 16 \\
\hline Gastronomie, Beherbergung & 18 & 23 & 27 & 9 & 5 & 8 \\
\hline Wellness, Friseure, Kosmetik & 17 & 15 & 19 & 10 & 7 & 11 \\
\hline Handwerk & 15 & 25 & 17 & 9 & 5 & 11 \\
\hline Vorrangig stationärer Handel & 13 & 28 & 14 & 23 & 11 & 32 \\
\hline Gesundheit, Medizin und Therapie & 11 & 24 & 16 & 35 & 15 & 28 \\
\hline Training/Coaching, Schulung/Unterricht & 10 & 31 & 15 & 59 & 22 & 36 \\
\hline Soziale Arbeit, Sozialwesen & 6 & 19 & 14 & 32 & 17 & 32 \\
\hline Durchschnitt & 37 & 48 & 34 & 27 & 15 & 22 \\
\hline
\end{tabular}

Anmerkung: Die dargestellten Werte geben den Anteil der befragten Soloselbständigen pro Branche an, deren Digitalisierungsgrad in den drei Bereichen vor der Corona-Pandemie (sehr) hoch war bzw. den Anteil der befragten Soloselbständigen, die den Digitalisierungsgrad seit Beginn der Corona-Pandemie erhöht haben. Je dunkler der Blauton (Grauton) umso höher (niedriger) ist der Anteil der befragten Soloselbständigen an allen Unternehmen der jeweiligen Branche.

Lesehilfe: $64 \%$ der befragten Soloselbständigen der Branche Onlinehandel geben an, dass der Digitalisierungsgrad ihrer Produkte oder Dienstleistungen (sehr) hoch ist und $9 \%$ haben den entsprechenden Digitalisierungsgrad seit Beginn der Corona-Pandemie erhöht.

Quelle: „Wie stark bist du als Selbständige/r von der Corona-Krise betroffen?“; Umfrage von VGSD, ZEW und der Universität Trier; Bertschek und Erdsiek $(2020,7)$.

Auch die Corona-Krise beflügelt Innovationen, wie aktuelle Zahlen der KfW für den deutschen Mittelstand zeigen (Zimmermann, 2020): Demnach haben $27 \%$ der Unternehmen zum Zeitpunkt der Befragung Innovationen Corona-bedingt umgesetzt und ihre Produkte, Prozesse oder Geschäftsmodelle erneuert oder verbessert, weitere $16 \%$ planen Innovationen aufgrund der Krise. Die meisten Innovatoren finden sich im Dienstleistungssektor und in kleinen Unternehmen, also insbesondere dort, wo stärkere Umsatzeinbußen zu verzeichnen waren. Dabei handelt es sich in erster Linie um Ad-hoc-Innovationen und selten um Innovationen, die aufwendige Forschungs- und Entwicklungsaktivitäten erfordern.

Die Corona-Krise hat also nicht nur die Digitalisierung der deutschen Wirtschaft beschleunigt, sondern auch zu deren Innovationsaktivität beigetragen, auch wenn diese sich zunächst auf einfach umsetzbare Maßnahmen bezieht. Werden diese Entwicklungen zu nachhaltigen Veränderungen führen oder sind sie lediglich ein Strohfeuer? Vieles wird von der weiteren Entwicklung der Infektionszahlen abhängen und davon, ob und wie lange die Maßnahmen des Konjunkturpakets greifen.

\section{Corona-bedingte Maßnahmen werden die Krise zum Teil überdauern}

Für die Nutzung des Homeoffice während der CoronaKrise haben $33 \%$ der Unternehmen in der Informationswirtschaft und $29 \%$ im verarbeitenden Gewerbe kurzfristig in neue Technologien investiert. Insgesamt $46 \%$ der Unternehmen in der Informationswirtschaft und 30\% im verarbeitenden Gewerbe gehen davon aus, dass sie auch nach der Krise vermehrt Homeoffice nutzen werden, und 
Abbildung 1

Unternehmen, deren Beschäftigte regelmäßig Homeoffice nutzen, vor, während und nach der Krise Anteil in \%

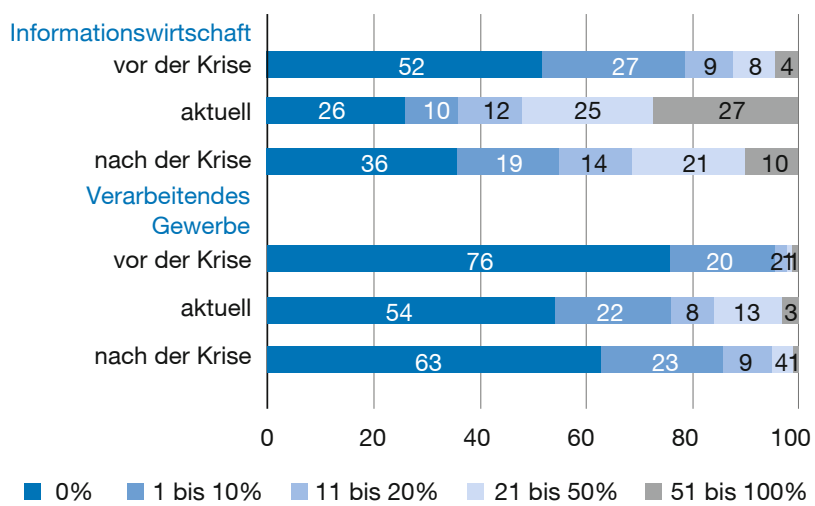

Lesehilfe: In $9 \%$ der Unternehmen in der Informationswirtschaft haben vor der Corona-Krise zwischen $11 \%$ und $20 \%$ der Beschäftigten regelmäßig mindestens einmal wöchentlich im Homeoffice gearbeitet. Nach der Krise soll dieser Unternehmensanteil bei schätzungsweise $14 \%$ liegen.

Quelle: ZEW Konjunkturumfrage Informationswirtschaft, 2. Quartal 2020, (ZEW, 2020b).

zwar in größerem Umfang als vor der Krise, aber in geringerem Umfang als während der Krise (ZEW, 2020, vgl. Abbildung 1). In der Informationswirtschaft gehen beispielsweise $31 \%$ der Unternehmen davon aus, dass mehr als ein Fünftel der Beschäftigten auch nach der Krise regelmäßig mindestens einmal wöchentlich Homeoffice praktizieren werden, d.h. weniger Unternehmen als während der Krise (52\%), aber deutlich mehr als vor der Krise (12\%). Insbesondere größere Unternehmen haben die Erfahrung gemacht, dass sich mehr Tätigkeiten im Homeoffice erledigen lassen als vor der Krise angenommen, und möchten daher auch nach der Krise eher am Homeoffice festhalten. Ein ähnliches Muster zeigt sich bei der Nutzung von Telefon- und Videokonferenzen.

Die Erwartungen zur konjunkturellen Entwicklung werden zunehmend besser, obwohl die Einschätzung der aktuellen Lage noch recht düster bleibt (ZEW-Konjunkturerwartungen Juli 2020, ZEW 2020a). Insbesondere für die Branche der Informations- und Kommunikationstechnologien (IKT), für die Dienstleister allgemein sowie für den Bau, erwarten die Finanzmarktexperten eine Verbesserung der Entwicklung. Die konjunkturelle Lage in der IKTBranche als zentraler Anbieter digitaler Technologien und Anwendungen hat im zweiten Quartal 2020 einen historischen Tiefstand erreicht (ZEW-Branchenreport, ZEW 2020b). Allerdings herrscht - zwar verhalten, aber mehrheitlich - Optimismus, was die zukünftige Entwicklung von Nachfrage und Umsatz angeht. Für das dritte Quartal
2020 rechnen rund $22 \%$ der IKT-Unternehmen mit Personalabbau, knapp $15 \%$ planen Neueinstellungen. Sollte der durch die Coronalkrise angestoßene Digitalisierungsschub langfristig zu Investitionen in Digitalisierungsprojekte führen, dann wird sich dies auch in einer positiven Entwicklung der IKT-Branche niederschlagen.

Bislang schöpft Deutschland die Potenziale der Digitalisierung nicht aus. Im EU-Vergleich liegt Deutschland auf Platz 18 von 28 bei der Nutzung digitaler Technologien in der Wirtschaft. Nur $6 \%$ der deutschen Unternehmen nutzen bislang Anwendungen der künstlichen Intelligenz. Die Corona-Krise hat zudem Defizite bei der digitalen Infrastruktur und im öffentlichen Bereich noch sichtbarer gemacht. So mangelt es in so manchem Haushalt, der sowohl Homeoffice als auch Homeschooling betreibt, an schnellem Internet, im Gesundheitswesen herrscht die Zettelwirtschaft.

\section{Das Zukunftspaket der Bundesregierung setzt auf Digitalisierung und Innovationen}

Das Konjunkturpaket der Bundesregierung ist in erster Linie auf das Abfedern der negativen Auswirkungen der Krise konzentriert. Hierzu zählen insbesondere das Kurzarbeitergeld, das nun bis Ende 2021 verlängert wurde, und die Staatsbeteiligung an „systemrelevanten“ Unternehmen. Mit dem als Zukunftspaket bezeichneten Teil des Konjunkturpakets hat die Bundesregierung ein Signal dafür gesetzt, dass die deutsche Wirtschaft in puncto Digitalisierung nach der Corona-Krise eine bessere Ausgangssituation haben soll als sie es vor der Krise hatte. So werden (zusätzliche) Mittel für den weiteren Ausbau von Glasfaser und 5G, die künstliche Intelligenz und die Quantentechnologie sowie für die Unterstützung der Wirtschaft und insbesondere der kleinen und mittleren Unternehmen bei der Digitalisierung bereitgestellt. Diese Maßnahmen wurden größtenteils bereits vor der CoronaKrise initiiert, haben aber durch die Krise an Bedeutung gewonnen. Jetzt gilt es dran zu bleiben:

1. Für eine zukunftsfähige digitale Infrastruktur müssen Genehmigungsverfahren für den Glasfaserausbau weiter vereinfacht werden.

2. Die seit längerem währende Diskussion über die Zulassung chinesischer Anbieter von Netzkomponenten für den Ausbau von 5G-Netzen muss in europaweit geltenden Sicherheitsstandards münden, die für alle Anbieter gelten (z. B. EFI, 2020a).

3. Für eine europäische Cloud-Infrastruktur wurde von Politik und Wirtschaft das Prestigeprojekt GAIA-X initiiert. Dies gilt es mit Nachdruck voranzutreiben oder, 
bei Absehen eines Scheiterns, möglichst schnell die Reißleine zu ziehen.

4. Die Entwicklung von Software, die Produkten und Diensten intelligente Funktionen verleiht, braucht Expertinnen und Experten. Jede zweite offene KI-Stelle konnte im vergangenen Jahr nicht besetzt werden (Rammer et al., 2020). Hier sind Investitionen in die Aus- und Weiterbildung gefragt.

5. Daten sind zum essenziellen „Produktionsfaktor" geworden. Die Bundesregierung hat sich zum Ziel gesetzt, ihre Datenstrategie in die EU-Ratspräsidentschaft einzubringen und an einer europäischen Lösung mitzuwirken. Dies ist nötig, um über die DSGVO hinaus einheitliche Rahmenbedingungen für den europäischen digitalen Binnenmarkt zu schaffen.

6. Digitale Technologien haben als "General Purpose Technologies" die Eigenschaft ihre Nutzer zu Innovationen zu befähigen (z. B. Bresnahan und Trajtenberg, 1995). Dieses Potenzial sollte mehr denn je genutzt und die Anreize für Innovationen verstärkt werden. Eine Maßnahme im Zukunftspaket betrifft die Erhöhung der förderfähigen Aufwendungen für die steuerliche Forschungszulage, die erst Anfang 2020 eingeführt wurde. Wie die Expertenkommission Forschung und Innovation (EFI, 2020b) darlegt, werden insbesondere größere Unternehmen in die Gunst dieser zusätzlichen Förderung kommen. Sie zeigt mögliche Alternativen auf, die kleinere und mittlere Unternehmen nicht benachteiligen.

7. Maßnahmen, die kleinere und mittlere Unternehmen bei der Digitalisierung unterstützen, wie sie durch verschiedene Förderprogramme schon vor der CoronaKrise gestartet wurden, müssen jetzt mit Nachdruck weitergeführt wird.

\section{Das Momentum nutzen}

Digitalisierung ist nicht um ihrer selbst willen so wichtig, sondern weil sie für viele Fragen und Herausforderungen eine Lösung bietet. Deshalb setzt das Zukunftspaket der Bundesregierung, das größtenteils zuvor bestehende Maßnahmen weiter verstärkt, die richtigen Signale.

Für Unternehmen heißt es nun, das Momentum zu nutzen und nicht bei Homeoffice und Videokonferenzen als neuen Errungenschaften zu verharren, sondern strategisch zu investieren, um die Eigenschaften, die sich seit Beginn der Corona-Krise als vorteilhaft erwiesen haben (Flexibilität, Distanzwahrung und Sichtbarkeit), auch langfristig zu sichern. Dabei ist es wichtig, sich die Digitalisierung für die (Weiter-)Entwicklung neuer Ideen zunutze zu machen. Die Krise hat zahlreiche neue Produkte und Dienste hervorgebracht oder bereits bestehenden zum Durchbruch verholfen. Denken wir beispielsweise an das veränderte Mobilitätsverhalten und die damit einhergehende groBe Nachfrage in der Fahrradbranche. Oder an den enormen Boom des Onlinehandels. Hier hat sich nicht nur die Nachfrage nach Produkten erhöht, die schon vor der Krise online vertrieben wurden, z. B. Bücher oder Elektrogeräte, sondern auch das Spektrum der gehandelten Produkte und Dienste. Mit Kochboxen werden nicht nur Nahrungsmittel online vertrieben, sondern Kocherlebnisse. Auch Dienste wie Coachings oder Weiterbildungskurse haben sich zügig in die Online-Welt verlagert. Auf diese Dynamik gilt es aufzusetzen und in die Entwicklung grundlegender Innovationen zu investieren.

Die Digitalisierung übernimmt in der Corona-Krise zweierlei Funktionen: Zum einen hilft sie dabei die negativen Auswirkungen der Krise abzufedern. Zum anderen beschleunigt sie den ohnehin bevorstehenden Strukturwandel, indem sie Innovationen ermöglicht.

\section{Literatur}

Bertschek, I. und D. Erdsiek (2020), Soloselbstständigkeit in der CoronaKrise; Digitalisierung hilft bei der Bewältigung der Krise, ZEW-Kurzexpertise, 20-8, Mannheim, ftp.zew.de/pub/zew-docs/ZEWKurzexpertisen/ZEW_Kurzexpertise2008.pdf (2. September 2020).

Bertschek, I., M. Polder und P. Schulte (2019), ICT and Resilience in Times of Crisis: Evidence from Cross-Country Micro Moments Data, Economics of Innovation and New Technology, 28(8), 759-774.

Bresnahan, T. und M. Trajtenberg (1995), General Purpose Technologies: Engines of Growth?, Journal of Econometrics, 65(1), 83-108.

EFI - Expertenkommission Forschung und Innovation (2020a), Gutachten zu Forschung, Innovation und technologischer Leistungsfähigkeit 2020, Berlin.

EFI - Expertenkommission Forschung und Innovation (2020b), Kleine und mittlere Unternehmen unterstützen, Forschungszulage an die Erfordernisse der Corona-Krise anpassen, EFI Policy Brief, Nr. 4-2020, Berlin.

Rammer, C., I. Bertschek, B. Schuck, V. Demary und H. Goecke (2020), Einsatz von Künstlicher Intelligenz in der Deutschen Wirtschaft, Stand der KI-Nutzung im Jahr 2019, Bundesministerium für Wirtschaft und Energie (BMWi), Berlin.

Schwartz, M. und J. Gerstenberger (2020), Corona-Krise hat den Mittelstand fest im Griff, aber Widerstandsfähigkeit (noch) hoch, KfW Research, Fokus Volkswirtschaftslehre, Nr. 286, 28. April 2020.

ZEW (2020a), ZEW-Konjunkturerwartungen Juli 2020, https://www.zew. de/presse/pressearchiv/leichter-rueckgang-der-erwartungen (9. September 2020).

ZEW (2020b), ZEW-Branchenreport Informationswirtschaft, 2. Quartal 2020, ftp.zew.de/pub/zew-docs/brepikt/202002BreplKT.pdf (2. September 2020).

Zimmermann, V. (2020), Innovationen in der Corona-Krise: Not macht erfinderisch, KfW Research, Fokus Volkswirtschaftslehre, Nr. 295, 13. Juli 2020 . 